\title{
Spin-orbit couplings between distant electrons trapped individually on liquid Helium
}

\author{
M. Zhang ${ }^{1}$ and L. F. Wei ${ }^{* 1,2}$ \\ ${ }^{1}$ Quantum Optoelectronics Laboratory, School of Physics, \\ Southwest Jiaotong University, Chengdu 610031, China \\ ${ }^{2}$ State Key Laboratory of Optoelectronic Materials and Technologies, \\ School of Physics and Engineering, Sun Yat-sen University, Guangzhou 510275, China
}

(Dated: November 10, 2018)

\begin{abstract}
We propose an approach to entangle spins of electrons floating on the liquid Helium by coherently manipulating their spin-orbit interactions. The configuration consists of single electrons, confined individually on liquid Helium by the micro-electrodes, moving along the surface as the harmonic oscillators. It has been known that the spin of an electron could be coupled to its orbit (i.e., the vibrational motion) by properly applying a magnetic field. Based on this single electron spin-orbit coupling, here we show that a Jaynes-Cummings (JC) type interaction between the spin of an electron and the orbit of another electron at a distance could be realized via the strong Coulomb interaction between the electrons. Consequently, the proposed JC interaction could be utilized to realize a strong orbit-mediated spin-spin coupling and implement the desirable quantum information processing between the distant electrons trapped individually on liquid Helium.

PACS numbers: 73.20.-r, 03.67.Lx, 33.35.+r
\end{abstract}

\footnotetext{
*weilianfu@gmail.com
} 


\section{INTRODUCTION}

The interactions between the microscopic particles, e.g., the ions in Paul trap [1], the neutral atoms confined in optical lattice [2], and the electrons in Penning trap [3], etc., relate usually to their masses and the inter-particle forces. Due to the small mass and the strong Coulomb interaction, the interacting electrons could be used to implement quantum information processing (QIP). The idea of quantum computing with strongly-interacting electrons on liquid Helium was first proposed by Platzman and Dykman in 1999 [4]. In their proposal, the two lower hydrogen-like levels of the surface-state electron are encoded as a qubit, and the effectively interbit couplings can be realized by the electric dipole-dipole interaction. When the liquid helium is cooled on the order of $\mathrm{mK}$ temperature the qubit possesses long coherent time (e.g., up to the order of ms) [5, 6]. Interestingly, Lyon suggested [7] that the qubits could also be encoded by the spins of the electrons on liquid Helium, and estimated that the qubit coherent time could reach $100 \mathrm{~s}$ [7]. He showed further that the magnetic dipole-dipole interactions between the spins could be used to couple the qubits, if the electrons are confined closed enough. For example, the coupling strength can reach to the

order of $\mathrm{kHz}$ for the distance $d=0.1 \mu \mathrm{m}$ between the electrons [7]. Remarkably, recent experiments [8-10] demonstrated the manipulations of electrons (confining, transporting, and detecting) on liquid Helium in the single-electron regime. This provides really the experimental platforms to realize the relevant QIP with electrons on liquid Helium [11-14].

Here, we propose an alternative approach to implement QIP with electronic spins on liquid Helium by coherently manipulating the spin-orbit interactions of the electrons. In our proposal, the virtues of long-lived spin states (to encode the qubit) and strong Coulomb interaction (for realizing the expectably-fast interbit operations) are both utilized. The electrons are trapped individually on the surface of liquid Helium by the micro-electrodes. In the plane of liquid Helium surface each electron moves as a harmonic oscillator. It has been showed that such an external orbit-vibration could be effectively coupled to the internal spin of a single electron by applying a magnetic field with a gradient along the vibrational axis [13]. Interestingly, we show that the spin of an electron could be coupled to the vibrational motion of another distant electron [as a Jaynes-Cummings (JC) type interaction], by designing a proper virtual excitation of the electronic vibration. The present JC interaction could be utilized to significantly enhance the spin-spin coupling between the distant electrons, and implement the desirable quantum computation with the spin qubits on liquid Helium. 


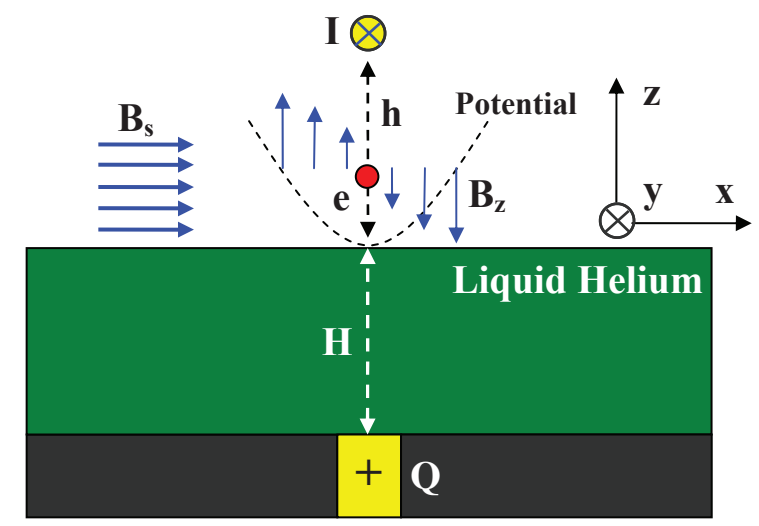

FIG. 1: (Color online) Sketch for a single electron trapped on the surface of liquid Helium. The liquid Helium provides $z$-directional confinement, and the micro-electrode Q (below the Helium surface at depth $H$ ) traps the electron in $x-y$ plane. The desirable spin qubit is generated by an applied uniform magnetic field $B_{s}$, and the spin-orbit coupling of the trapped electron is obtained by applying a current to another micro-electrode I (upon the liquid Helium surface at the height $h$ ).

The paper is organized as follows: In Sec. II we discuss the mechanism for spin-orbit coupling with a single electron trapped on liquid Helium [13], and then show how to utilize such a coupling to realize the desirable quantum gate with the single electron. By using the electron-electron Coulomb interaction, in Sec. III, we propose an approach to implement the JC coupling between the spin of an electron and the orbital motion of another electron. Based on such a distant spinorbit interaction, we show that a two-qubit controlled-NOT (CNOT) gate and an orbit-enhanced coupling between the distant spins could be implemented. Finally, we give a conclusion in Sec. IV.

\section{SPIN-ORBIT COUPLING WITH A SINGLE TRAPPED ELECTRON}

We consider first a single electron trap shown in Fig. 1 [13], wherein an electron (with mass $m_{e}$ and charge $e$ ) on liquid Helium is weakly attracted by its dielectric image potential $V(z)=$ $-\Lambda e^{2} / z$ (with $\Lambda=(\varepsilon-1) / 4(\varepsilon+1)$ and $\varepsilon$ being the dielectric constant of liquid Helium). Due to the Pauli exclusion principle, there is an barrier (about $1 \mathrm{eV}$ ) to prevent the electron penetrating into the liquid Helium. As a consequence, z-directional confinement of the electron is realized, yielding an one-dimensional (1D) hydrogenlike atom with the spectrum $E_{n}=-\hbar R / n^{2}$ [15]. Here, $R=\Lambda^{2} e^{4} m_{e} /\left(2 \hbar^{2}\right) \approx 170 \mathrm{GHz}$ and $r_{\mathrm{b}}=\hbar^{2} /\left(m_{e} e^{2} \Lambda\right) \approx 7.6 \mathrm{~nm}$ are the effective Rydberg 
energy and Bohr radius, respectively. In $x-y$ plane, the electron can be confined by the microelectrode $\mathrm{Q}$ located at $H$ beneath the liquid Helium surface. Typically, $x, y, z \ll H$, and thus the potential of the electron can be described by [5]

$$
U(x, y, z) \approx-\frac{\Lambda e^{2}}{z}+E_{\perp} z+\frac{m_{e}}{2}\left(\nu_{x}^{2} x^{2}+\nu_{y}^{2} y^{2}\right)
$$

with $E_{\perp}=e Q / H^{2}, \nu_{x}=\nu_{y}=\sqrt{e Q /\left(m_{e} H^{3}\right)}$, and $Q$ being the effective charge of the microelectrode. This potential indicates that the motions of the trapped electron are a 1D Stark-shifted hydrogen along the $z$-direction, and a 2D harmonic oscillator in the plane parallel to the liquid Helium surface. The Hamiltonian for the orbital motions of the trapped electron can be written as

$$
\hat{H}_{o}=\sum_{n} E_{n}\left|n_{a}\right\rangle\left\langle n_{a}\right|+\sum_{k=x, y} \hbar \nu_{k}\left(\hat{a}_{k}^{\dagger} \hat{a}_{k}+\frac{1}{2}\right) .
$$

Here, $\left|n_{a}\right\rangle$ is the $n$th bound state of the hydrogenlike atom, $\hat{a}_{k}^{\dagger}$ and $\hat{a}_{k}$ are the bosonic operators of the vibrational quanta of the electron along the $k$-direction.

A spin qubit is generated by applying an uniform magnetic field $B_{s}$ along $x$ direction, and its Hamiltonian reads $\hat{H}_{q}=\left(g \mu_{B} B_{s}\right) \hat{\sigma}_{x} / 2$. Here, the Pauli operator is defined as $\hat{\sigma}_{x}=|\uparrow\rangle\langle\uparrow$ $|-| \downarrow\rangle\langle\downarrow|$ with $|\downarrow\rangle$ and $|\uparrow\rangle$ being the two spin states. $g=2$ is the electronic $g$-factor, and $\mu_{B}=9.3 \times 10^{-24} \mathrm{~J} / \mathrm{T}$ is the Bohr magneton. The spin-orbit coupling of the trapped electron can be realized by applying a dc current $I$ to the electrode I (located upon the liquid Helium surface with a height $h$ ) [13]. Typically, $x, z \ll h$ and the magnetic field generated by the current $I$ reads $\vec{B}=\left(B_{x}, 0, B_{z}\right)$ with $B_{x} \approx \mu_{0} I(1-z / h) /(2 \pi h)$ and $B_{z} \approx \mu_{0} I x /\left(2 \pi h^{2}\right)$. Here, $\mu_{0}$ is the permeability of free space. Therefore, the Hamiltonian describing the interaction between the magnetic field and spin can be expressed as: $\hat{H}_{\mathrm{sb}}=g \mu_{B}\left(B_{z} \hat{\sigma}_{z}+B_{x}^{\prime} \hat{\sigma}_{x}\right) / 2$ with $B_{x}^{\prime}=B_{s}+B_{x}$, $\hat{\sigma}_{z}=\hat{\sigma}_{-}+\hat{\sigma}_{+}, \hat{\sigma}_{-}=|\downarrow\rangle\langle\uparrow|$ and $\hat{\sigma}_{+}=|\uparrow\rangle\langle\downarrow|$. Consequently, the total Hamiltonian of the trapped electron in the applied magnetic fields reads

$$
\hat{H}=\frac{\hbar \nu_{s}}{2} \hat{\sigma}_{x}+\hat{H}_{o}+\hat{H}_{s x}
$$

with

$$
\hat{H}_{s x}=\frac{g \mu_{B} \mu_{0} I}{4 \pi h^{2}} \sqrt{\frac{\hbar}{2 m_{e} \nu_{x}}}\left(\hat{a}_{x}+\hat{a}_{x}^{\dagger}\right) \hat{\sigma}_{z} .
$$

The first and second terms in the right hand of Eq. (3) describe the free Hamiltonian of the trapped electron, with $\nu_{s}=\left(g \mu_{B} / \hbar\right)\left[B_{s}+\left(\mu_{0} I / 2 \pi h\right)\right]$ being the transition frequency between its two spin states, and $\hat{H}_{s x}$ describes the coupling between the spin and the orbital motion along $x$-direction. 
Note that the coupling between the spin and $z$-directional orbital motion is neglected, due to the large-detuning. Also, the applied strong field $B_{s}$ (e.g., $0.06 \mathrm{~T}$ ) does not affect the interaction $\hat{H}_{s x}$, although it will change slightly the electron's motions in the $y$ - $z$ plane [16].

Obviously, the Hamiltonian in Eq. (3) can be simplified as

$$
\hat{H}_{e}=\hbar \Omega\left(e^{i \delta t} \hat{\sigma}_{+} \hat{a}+e^{-i \delta t} \hat{\sigma}_{-} \hat{a}^{\dagger}\right)
$$

in the interaction picture. Here, $\delta=\nu_{s}-\nu_{x}$ is the detuning,

$$
\Omega=\frac{g \mu_{B} \mu_{0} I}{4 \pi h^{2} \sqrt{2 \hbar m_{e} \nu_{x}}}
$$

is the coupling strength, and $\hat{a}=\hat{a}_{x}, \hat{a}^{\dagger}=\hat{a}_{x}^{\dagger}$. Note that, the Hamiltonian in Eq. (5) can also be obtained by applying an ac current $I(t)=I \cos (\omega t)$ with frequency $\omega=\nu_{x}-\nu_{s}+\delta$ to the electrode. Specially, when $\delta=0$, this Hamiltonian describes a JC-type interaction between the spin and orbit of the single electron. In fact, Ref. [13] has arranged this spin-orbit coupling of a single electron to increase the interaction between the spin and a quantized microwave field. Alternatively, we will utilize this spin-orbit coupling (together with the electron-electron strong Coulomb interaction) to realize a strong interaction between two electronic spins and generate certain typical quantum gates.

For the typical parameters: $I=1 \mathrm{~mA}, h=0.5 \mu \mathrm{m}$, and $\nu_{x}=10 \mathrm{GHz}[15$, , 13], we have $\Omega \approx 5.2 \mathrm{MHz}$. This is significantly larger than the decoherence rate (which is typically on the order of $10 \mathrm{kHz}$ [5, 13]) of the vibrational states of the trapped electron. Thus, the above JC interaction provides a possible approach to implement QIP between the spin and orbit states of a single trapped electron. For the JC interaction, the state-evolutions can be limited in the invariant-subspaces $\{\mid \downarrow$ $, 0\rangle\}$ and $\{|\downarrow, 1\rangle,|\uparrow, 0\rangle\}$, with $|0\rangle$ and $|1\rangle$ being the ground and first excited states of the harmonic oscillator. Thus, a phase gate $\hat{P}=|0, \downarrow\rangle\langle 0, \downarrow|+| 0, \uparrow\rangle\langle 0, \uparrow|+| 1, \downarrow\rangle\langle 1, \downarrow|-| 1, \uparrow\rangle\langle 1, \uparrow|$ could be implemented by applying a current pulse to the electrode I. The relevant duration $t$ is set to satisfy the conditions: $\sin (\Omega t) \approx 0$ and $\cos (\sqrt{2} \Omega t) \approx-1$ (e.g., $\Omega t \approx 37.7$ numerically). Consequently, a CNOT gate with the single electron could be realized as $\hat{S}=\hat{R}(\pi / 2,-\pi / 2) \hat{P} \hat{R}(\pi / 2, \pi / 2)$, where $\hat{R}(\alpha, \beta)=(|\uparrow\rangle\langle\uparrow|+| \downarrow\rangle\langle\downarrow|) \cos (\alpha)-i[\exp (i \beta)|\uparrow\rangle\langle\downarrow|+\exp (-i \beta)| \downarrow\rangle\langle\uparrow|] \sin (\alpha)$ is an arbitrary single-bit rotation [17]. This CNOT gate operation, between the spin states and the two selected vibrational states of a single electron [18], is an intermediate step for the later CNOT operation between two distant spin qubits. 


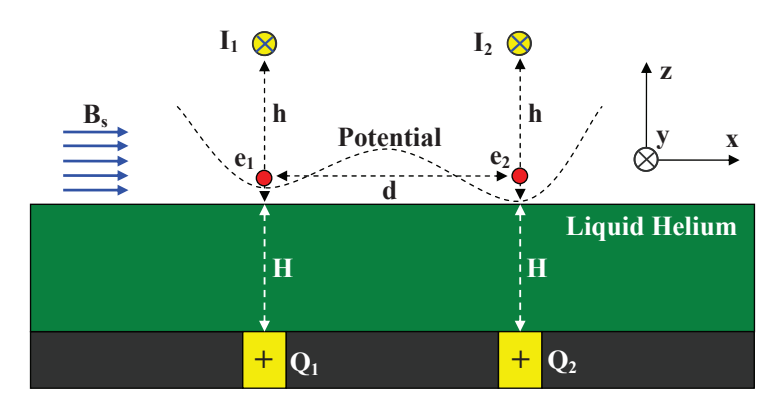

FIG. 2: (Color online) Two electrons (denoted by $e_{1}$ and $e_{2}$ ) are confined individually in two potential wells with the distance $d$, which is sufficiently large (e.g., $d=10 \mu \mathrm{m}$ ) such that the magnetic dipoledipole coupling between the electronic spins is negligible. The orbital motions of the two electrons are also decoupled from each other, since they are trapped in large-detuning regime. By applying a current to the electrode $\mathrm{I}_{1}$ the spin of the electron $e_{1}$ could be coupled to the vibrational motions of electron $e_{2}$, via a virtual excitation of the vibrational motion of electron $e_{1}$.

\section{SPIN-ORBIT JC COUPLING BETWEEN THE DISTANT ELECTRONS}

Without loss of generality, we consider here two electrons (denoted by $e_{1}$ and $e_{2}$ ) trapped individually in two potential wells, see Fig. 2. Suppose that the distance $d$ between the potential wells is sufficiently large (e.g., $d=10 \mu \mathrm{m}$ ), such that the directly magnetic interaction between the two spins could be neglected. Thus, the interaction between the two electrons leaves only the Coulomb one. Specially, the Coulomb interaction along the $x$-direction can be approximately written as

$$
V(x) \approx \frac{e^{2}}{2 \pi \epsilon_{0} d^{3}} x_{1} x_{2}
$$

with $x_{j}$ being the displacement of electron $e_{j}$ from its potential minima. By controlling the voltages applied on the electrodes $Q_{1}$ and $Q_{2}$, the vibrational frequencies of the electrons are set as the large-detuning (and thus the electrons are decoupled from each other).

To couple the initially-decoupled electrons, we apply a current $I$ to the electrode $\mathrm{I}_{1}$. As discussed previously, such a current induces a spin-orbit coupling [i.e., $\hat{H}_{e}$ in Eq. (5)] of the electron $e_{1}$. Therefore, the present two-electrons system can be described by the following Hamiltonian

$$
\hat{H}_{e e}=\hat{H}_{e}+\hbar \tilde{\Omega}\left(e^{i \Delta t} \hat{a} \hat{b}^{\dagger}+e^{-i \Delta t} \hat{a}^{\dagger} \hat{b}\right)
$$

in the interaction picture. Where, $\hat{b}$ and $\hat{b}^{\dagger}$ are the bosonic operators of the vibrational motion of electron $e_{2}$ along $x$-direction, $\Delta=\nu_{2 x}-\nu_{1 x}$ is the detuning between the two electronic vibrations 
along $x$-direction, and

$$
\tilde{\Omega}=\frac{e^{2}}{4 \pi \epsilon_{0} m_{e} d^{3} \sqrt{\nu_{1 x} \nu_{2 x}}},
$$

the coupling strength. Numerically, for $d=10 \mu \mathrm{m}$ and $\nu_{j x}=10 \mathrm{GHz}$ we have $\tilde{\Omega} \approx 25 \mathrm{MHz}$. Above, the spin of electron $e_{2}$ was dropped, as the driving (induced by electrode $\mathrm{I}_{1}$ ) on this spin is negligible (due to $d \gg h$ ).

The dynamical evolution ruled by the Hamiltonian in Eq. (8) is given by the following timeevolution operator

$$
\begin{aligned}
\hat{U}(t)= & +\left(\frac{-i}{\hbar}\right) \int_{0}^{t} \hat{H}_{e e}\left(t_{1}\right) d t_{1} \\
& +\left(\frac{-i}{\hbar}\right)^{2} \int_{0}^{t} \hat{H}_{e e}\left(t_{1}\right) \int_{0}^{t_{1}} \hat{H}_{e e}\left(t_{2}\right) d t_{2} d t_{1}+\cdots
\end{aligned}
$$

We assume $\delta=\Delta$ for simplicity, then the above time-evolution operator can be approximated as

$$
\hat{U}(t) \approx \exp \left(-\frac{i t}{\hbar} \hat{H}_{\mathrm{eff}}\right)
$$

with the effective Hamiltonian

$$
\begin{aligned}
\hat{H}_{\mathrm{eff}}=\frac{\hbar \Omega^{2}}{\delta} & {\left[\hat{a}^{\dagger} \hat{a}\left(\hat{\sigma}_{+} \hat{\sigma}_{-}-\hat{\sigma}_{-} \hat{\sigma}_{+}\right)+\hat{\sigma}_{+} \hat{\sigma}_{-}\right] } \\
& +\frac{\hbar \tilde{\Omega}^{2}}{\delta}\left(\hat{b}^{\dagger} \hat{b}-\hat{a}^{\dagger} \hat{a}\right)+\frac{\hbar \Omega \tilde{\Omega}}{\delta}\left(\hat{\sigma}_{+} \hat{b}+\hat{\sigma}_{-} \hat{b}^{\dagger}\right) .
\end{aligned}
$$

The second term in the right hand of Eq. (10) and the terms relating to the high orders of $\Omega / \delta$ and $\tilde{\Omega} / \delta$ were neglected, since $\Omega, \tilde{\Omega} \ll \delta$. Furthermore, at the experimental temperature (e.g., $20 \mathrm{mK}$ ) the electrons are frozen well into their vibrational ground states (about $40 \mathrm{mK}$ for the vibrational frequency $\sim 10 \mathrm{GHz}$ ). This means that the excitation of the vibration of electron $e_{1}$ is virtual, and thus the terms in Eq. (12) related to $\hat{a}^{\dagger} \hat{a}$ can be adiabatically eliminated. As a consequence, the Hamiltonian in Eq. (12) reduces to

$$
\hat{H}_{\mathrm{eff}}=\frac{\hbar \Omega^{2}}{\delta} \hat{\sigma}_{+} \hat{\sigma}_{-}+\frac{\hbar \tilde{\Omega}^{2}}{\delta} \hat{b}^{\dagger} \hat{b}+\frac{\hbar \Omega \tilde{\Omega}}{\delta}\left(\hat{\sigma}_{+} \hat{b}+\hat{\sigma}_{-} \hat{b}^{\dagger}\right)
$$

and further reads (for $\Omega=\tilde{\Omega}$ )

$$
\hat{H}_{\mathrm{JC}}=\frac{\hbar \Omega^{2}}{\delta}\left(\hat{\sigma}_{+} \hat{b}+\hat{\sigma}_{-} \hat{b}^{\dagger}\right)
$$

in the interaction picture. Obviously, this Hamiltonian describes a JC-type coupling between the spin of electron $e_{1}$ and the orbital motion of electron $e_{2}$. 


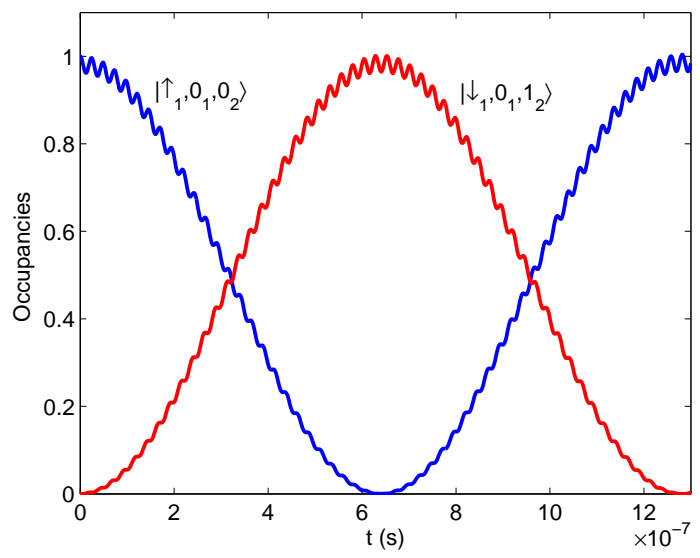

FIG. 3: (Color online) Numerical solutions for the Hamiltonian in Eq. (8): the occupancy evolutions of states $\left|\uparrow_{1}, 0_{1}, 0_{2}\right\rangle$ (blue curve) and $\left|\downarrow_{1}, 0_{1}, 1_{2}\right\rangle$ (red curve), with $\tilde{\Omega}=\Omega=25 \mathrm{MHz}$ and $\delta=\Delta=250 \mathrm{MHz}$.

Typically, the effective coupling strength can reach $\Omega^{\prime}=\Omega^{2} / \delta \approx 2.5 \mathrm{MHz}$ for $d=10 \mu \mathrm{m}$, $\nu_{1 x}=10 \mathrm{GHz}$, and $\delta=250 \mathrm{MHz}$. With these parameters and the Hamiltonian in E.q (8), Fig. 3 shows numerically the occupancy evolutions of the states $\left|\uparrow_{1}, 0_{1}, 0_{2}\right\rangle$ and $\left|\downarrow_{1}, 0_{1}, 1_{2}\right\rangle$. Here, $\left|\downarrow_{j}\right\rangle$ and $\left|\uparrow_{j}\right\rangle$ are the two spin states of electron $e_{j}$, and $\left|0_{j}\right\rangle$ and $\left|1_{j}\right\rangle$ are the two lower vibrational states of the electron. Obviously, the results are well agreement with the solutions (i.e., the timedependent occupancies of $\left|\uparrow_{1}, 0_{2}\right\rangle$ and $\left.\left|\downarrow_{1}, 1_{2}\right\rangle\right)$ from the Hamiltonian $\hat{H}_{\mathrm{JC}}$. This verifies the validity of $\hat{H}_{\mathrm{JC}}$. The spin-orbit JC coupling (14) could be used to implement QIP between the separately trapped electrons. For example, by applying a current pulse with the duration $t=\pi /\left(2 \Omega^{\prime}\right)$ to an electrode, e.g., $\mathrm{I}_{1}$, a two-qubit operation $\hat{V}_{1,2}(\pi / 2)=\left|\downarrow_{1}, 0_{2}\right\rangle\left\langle\downarrow_{1}, 0_{2}|-i| \downarrow_{1}, 1_{2}\right\rangle\left\langle\uparrow_{1}, 0_{2}\right|$ between the electrons could be implemented. Consequently, a CNOT gate between the qubits encoded by the electronic spins could be implemented by the operational sequence $\hat{C}=\hat{V}_{1,2}(\pi / 2) \hat{S}_{2} \hat{V}_{1,2}(\pi / 2)$, with $\hat{S}_{2}$ being the single-electron CNOT gate operated on the electron $e_{2}$. After this two-spin CNOT operation, the vibrational motions of the trapped electrons return to their initial ground states.

Furthermore, the mechanism used above for the distant spin-orbit coupling can be utilized to implement an orbit-mediated spin-spin interaction, wherein the degrees freedom of the orbits of the two electrons are adiabatically eliminated. Indeed, by applying the current pulses to the electrodes simultaneously, the Hamiltonian of the individually-driven electrons reads:

$$
\hat{H}_{e e}^{\prime}=\hbar \Omega\left(e^{i \delta t} \hat{\sigma}_{+} \hat{a}+e^{-i \delta t} \hat{\sigma}_{-} \hat{a}^{\dagger}\right)+\hbar \tilde{\Omega}\left(e^{i \delta t} \hat{a} \hat{b}^{\dagger}+e^{-i \delta t} \hat{a}^{\dagger} \hat{b}\right)+\hbar G\left(e^{i \eta t} \hat{\tau}_{+} \hat{b}+e^{-i \eta t} \hat{\tau}_{-} \hat{b}^{\dagger}\right)
$$




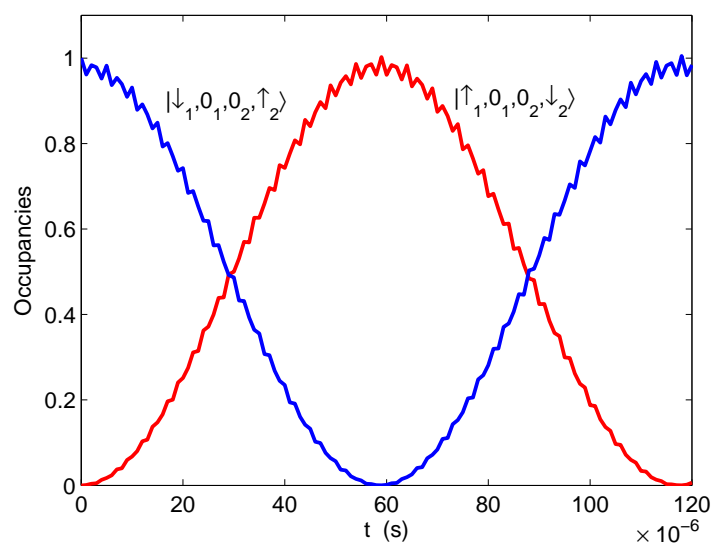

FIG. 4: (Color online) Numerical solutions for the Hamiltonian in Eq. (15): the occupancy evolutions of the states $\left|\downarrow_{1}, 0_{1}, 0_{2}, \uparrow_{2}\right\rangle$ (blue curve) and $\left|\uparrow_{1}, 0_{1}, 0_{2}, \downarrow_{2}\right\rangle$ (red curve), with $\tilde{\Omega}=25 \mathrm{MHz}, \Omega=2.6 \mathrm{MHz}$, $\delta=250 \mathrm{MHz}$, and $\eta=\Omega^{2} / \delta$.

Here, the first and third terms describe respectively the spin-orbit couplings of the electrons $e_{1}$ and $e_{2}$, and the second term describes the Coulomb interaction between the electrons. $G$ and $\eta$ are the coupling strength and the detuning between the spin and orbital motions of electron $e_{2}$, respectively. $\hat{\tau}_{-}=\left|\downarrow_{2}\right\rangle\left\langle\uparrow_{2}\right|$ and $\hat{\tau}_{+}=\left|\uparrow_{2}\right\rangle\left\langle\downarrow_{2}\right|$ are the corresponding spin operators of electron $e_{2}$. The spin-orbit couplings, i.e., the first and third terms in the Hamiltonian, can be realized by applying the ac currents $I_{1}(t)=I_{1} \cos \left(\omega_{1} t\right)$ and $I_{2}(t)=I_{2} \cos \left(\omega_{2} t\right)$ to the electrodes $\mathrm{I}_{1}$ and $\mathrm{I}_{2}$ respectively, with the frequencies $\omega_{1}=\nu_{1 x}-\nu_{s}+\delta$ and $\omega_{2}=\nu_{2 x}-\nu_{s}+\eta$. Here the ac currents are applied to relatively-easily satisfy the above requirements for the detunings.

With the help of Eq. (13), Eq. (15) can be effectively simplified as

$$
\hat{H}_{e e}^{\prime}=\hat{H}_{\mathrm{eff}}+\hbar G\left(e^{i \eta t} \hat{\tau}_{+} \hat{b}+e^{-i \eta t} \hat{\tau}_{-} \hat{b}^{\dagger}\right)
$$

i.e.,

$$
\hat{H}_{e e}^{\prime}=\frac{\hbar \Omega \tilde{\Omega}}{\delta}\left(e^{i \gamma t} \hat{\sigma}_{+} \hat{b}+e^{-i t \gamma} \hat{\sigma}_{-} \hat{b}^{\dagger}\right)+\hbar G\left(e^{i\left(\eta-\tilde{\Omega}^{2} / \delta\right) t} \hat{\tau}_{+} \hat{b}+e^{-i\left(\eta-\tilde{\Omega}^{2} / \delta\right) t} \hat{\tau}_{-} \hat{b}^{\dagger}\right)
$$

in the interaction picture, with $\gamma=\left(\Omega^{2}-\tilde{\Omega}^{2}\right) / \delta$. We select $G=\Omega \tilde{\Omega} / \delta$ and $\eta=\Omega^{2} / \delta$ for simplicity, such that

$$
\hat{H}_{e e}^{\prime}=\hbar G\left(e^{i \gamma t} \hat{\sigma}_{+} \hat{b}+e^{-i t \gamma} \hat{\sigma}_{-} \hat{b}^{\dagger}\right)+\hbar G\left(e^{i \gamma t} \hat{\tau}_{+} \hat{b}+e^{-i \gamma t} \hat{\tau}_{-} \hat{b}^{\dagger}\right)
$$

By repeating the same method for deriving the effective Hamiltonian $\hat{H}_{\text {eff }}$, i.e., neglecting the terms relating to the high orders of $G / \gamma$ in the time-evolution operator and eliminating adiabatically the 
terms relating to $\hat{b}^{\dagger} \hat{b}$, we have

$$
\hat{H}_{\text {eff }}^{\prime}=\frac{\hbar G^{2}}{\gamma}\left(\hat{\sigma}_{+} \hat{\tau}_{-}+\hat{\sigma}_{-} \hat{\tau}_{+}\right)
$$

This is an effectively interaction between the two spins, mediated by their no-excited orbital motions [19].

Numerically, for $\tilde{\Omega} \approx 25 \mathrm{MHz}, \Omega \approx 2.6 \mathrm{MHz}$, and $\delta \approx 250 \mathrm{MHz}$, we have $|\gamma| \approx 2.5 \mathrm{MHz}$, $G \approx 0.26 \mathrm{MHz}$, and $\Omega^{\prime \prime}=\left|G^{2} / \gamma\right| \approx 27 \mathrm{kHz}$. With these parameters, Fig. 4 shows numerically the time-dependent occupancies of $\left|\downarrow_{1}, 0_{1}, 0_{2}, \uparrow_{2}\right\rangle$ and $\left|\uparrow_{1}, 0_{1}, 0_{2}, \downarrow_{2}\right\rangle$ from the Hamiltonian in Eq. (15). This provides the validity of the simplified Hamiltonian in Eq. (19). Obviously, the present orbit-mediated spin-spin coupling is significantly weaker than the above spin-orbit JC coupling (14) between the electrons, but still stronger than the directly magnetic dipole-dipole coupling (which is estimated as $\sim 10^{-3} \mathrm{~Hz}$ for the same distance) between the spins. Since the coherence time of the spin qubit is very long (e.g., could be up to minutes [7]), the orbit-mediated spinspin coupling demonstrated above could be utilized to generate the spins entanglement and thus implement the desirable QIP.

Finally, we would like to emphasize that, the considered double-trap configuration shown in Fig. 2 seems similarly to that of the recent ion-trap experiments $[20,21]$. There, two ions are confined in two potential wells separated by $40 \mu \mathrm{m}$ [20] (or $54 \mu \mathrm{m}$ [21]), and the ion-ion vibrational coupling $\hat{H}_{i i}=\hbar \tilde{\Omega}\left[\exp (i \Delta t) \hat{a} \hat{b}^{\dagger}+\exp (-i \Delta t) \hat{a}^{\dagger} \hat{b}\right]$ is achieved up to $\tilde{\Omega} \approx 10 \mathrm{kHz}$ [20] (or $\tilde{\Omega} \approx$ $7 \mathrm{kHz}$ [21]). The coupling between the ions was manipulated tunably by controlling the potential wells (via sweeping the voltages on the relevant electrodes) to adiabatically tune the oscillators into or out of resonance, i.e., $\Delta=0$ or $\Delta \gg \tilde{\Omega}[22]$, respectively. Instead, in the present proposal we suggested a JC-type coupling (and consequently an orbit-mediated spin-spin coupling) between the two separated electrons. Therefore, the operational steps for implementing the QIP should be relatively simple. More interestingly, here the electron-electron coupling strength $\tilde{\Omega}$ is significantly stronger (about $10^{3}$ times) than that between the trapped ions $\left(\mathrm{e} . \mathrm{g},{ }^{9} \mathrm{Be}^{+}[20]\right)$, since the mass of electron is much smaller than that of the ions.

\section{CONCLUSION}

We have suggested an approach to implement the QIP with electronic spins on liquid helium. Two long-lived spin states of the trapped electron were encoded as a qubit, and the strong Coulomb 
interaction between the electrons was utilized as the data bus. The spin-orbit JC coupling between the spin of an electron and the vibrational motion of another distant electron is generated by designing a virtual excitation of the electronic vibration. Such a distant spin-orbit interaction is further utilized to realize an orbit-mediated spin-spin coupling and implement the desirable quantum gates.

Compared with the ions in the Paul traps, here a feature is that the mass of the electron is much smaller than that of ions, and thus a strong Coulomb coupling up to $25 \mathrm{MHz}$ between the electrons could reached for a distance of $d=10 \mu \mathrm{m}$. Finally, the construction suggested here for implementing quantum computation with trapped electrons on the liquid helium should be scalable, and hopefully be feasible with current micro-scale technique.

Acknowledgements: This work was partly supported by the National Natural Science Foundation of China Grants No. 11204249, 11147116, 11174373, and 90921010, the Major State Basic Research Development Program of China Grant No. 2010CB923104, and the open project of State Key Laboratory of Functional Materials for Informatics.

[1] W. Paul, Rev. Mod. Phys. 62, 531 (1990); D. J. Wineland, C. Monroe, W. M. Itano, D. Leibfried, B. E. King, and D. M. Meekhof, J. Res. Natl. Inst. Stand. Technol. 103, 259 (1998); J. I. Cirac and P. Zoller, Phys. Rev. Lett. 74, 4091 (1995).

[2] O. Morsch and M. Oberthaler, Rev. Mod. Phys. 78, 179 (2006).

[3] L. S. Brown and G. Gabrielse, Rev. Mod. Phys. 58, 233 (1986); L. Lamata, D. Porras, and J. I. Cirac, Phys. Rev. A 81, 022301 (2010).

[4] P. M. Platzman and M. I. Dykman, Science 284, 1967 (1999).

[5] M. I. Dykman, P. M. Platzman, and P. Seddighrad, Phys. Rev. B 67, 155402 (2003).

[6] E. Collin, W. Bailey, P. Fozooni, P. G. Frayne, P. Glasson, K. Harrabi, M. J. Lea, and G. Papageorgiou, Phys. Rev. Lett. 89, 245301 (2002).

[7] S. A. Lyon, Phys. Rev. A 74, 052338 (2006).

[8] F. R. Bradbury, M. Takita, T. M. Gurrieri, K. J. Wilkel, K. Eng, M. S. Carroll, and S. A. Lyon, Phys. Rev. Lett. 107, 266803 (2011); K. Kono, Physics, 4, 110 (2011).

[9] G. Papageorgiou, P. Glasson, K. Harrabi, V. Antonov, E. Collin, P. Fozooni, P. G. Frayne, M. J. Lea, and D. G. Rees, Appl. Phys. Lett. 86, 153106 (2005); G. Sabouret, F. R. Bradbury, S. Shankar, J. A. 
Bert, and S. A. Lyon, Appl. Phys. Lett. 92, 082104 (2008).

[10] M. Koch, G. Auböck, C. Callegari, and W. E. Ernst, Phys. Rev. Lett. 103, 035302 (2009).

[11] M. Zhang, H. Y. Jia and L. F. Wei, Phys. Rev. A 80, 055801 (2009).

[12] M. Zhang, H. Y. Jia and L. F. Wei, Opt. Lett. 351686 (2010).

[13] D. I. Schuster, A. Fragner, M. I. Dykman, S. A. Lyon, and R. J. Schoelkopf, Phys. Rev. Lett. 105, 040503 (2010).

[14] S. Mostame and R. Schützhold, Phys. Rev. Lett. 101, 220501 (2008).

[15] C. C. Grimes and T. R. Brown, Phys. Rev. Lett. 32, 280 (1974); D. Konstantinov, M. I. Dykman, M. J. Lea, Y. Monarkha, and K. Kono, Phys. Rev. Lett. 103, 096801 (2009).

[16] S. S. Sokolov, Phys. Rev. B 51, 2640 (1995).

[17] Alternatively, the single-bit operations could be implemented by applying an ac current $I(t)$ (with frequency $\omega=\nu_{s}$ and phase $\theta$ on the electrode $\mathrm{I}_{2}$, see, Fig. 2), which generates a magnetic field $B_{z} \approx \mu_{0} I(t) d /\left[2 \pi\left(d^{2}+h^{2}\right)\right]$ along the $z$-direction to excite resonantly the spin of electron $e_{1}$.

[18] C. Monroe, D. M. Meekhof, B. E. King, W. M. Itano, and D. J. Wineland, Phys. Rev. Lett. 754714 (1995).

[19] K. Mølmer and A. Sørensen, Phys. Rev. Lett. 821835 (1999).

[20] K. R. Brown, C. Ospelkaus, Y. Colombe, A. C. Wilson, D. Leibfried, and D. J. Wineland, Nature (London) 471, 196 (2011).

[21] M. Harlander, R. Lechner, M. Brownnutt, R. Blatt, and W. Hänsel, Nature (London) 471, 200 (2011).

[22] M. Zhang and L. F. Wei, Phys. Rev. A, 83064301 (2011). 